\title{
A NEW SUFFICIENT CONDITION FOR PERIODIC SOLUTIONS OF WEAKLY NONLINEAR DIFFERENTIAL SYSTEMS
}

L. CESARI AND J. K. HALE

1. We shall deal here with systems of differential equations of the form

$$
\ddot{y}_{i}+\sigma_{j}^{2} y_{j}=\epsilon f_{j}\left(y_{1}, \cdots, y_{n}, \dot{y}_{1}, \cdots, \dot{y}_{n}, \epsilon, t\right), \quad j=1,2, \cdots, n,
$$

where $\epsilon$ is a small real parameter, $\sigma_{1}, \cdots, \sigma_{n}$ are real positive numbers, and each $f_{j}$ is a real valued function periodic in the real variable $t$ of period $2 \pi / \omega, \omega>0$. In a previous paper R. A. Gambill and J. K. Hale [6] have given sufficient conditions for the existence of periodic solutions of (1) (and more general systems), whose dominant terms have periods in a rational ratio with $2 \pi / \omega$ (harmonics, subharmonics, ultra-subharmonics). Also, a number of examples and applications were given in [6]. The aim of the present paper is to prove a new general statement which contains as particular cases two of the various theorems proved in [6].

We shall use exactly the same method used in [6]. This method has been successively developed by L. Cesari, J. K. Hale and R. A. Gambill, in a series of papers concerning boundedness of solutions of linear differential systems with periodic coefficients $[1 ; 4 ; 5 ; 8]$, cycles of autonomous weakly nonlinear differential systems [9], and harmonics and subharmonics of periodic weakly nonlinear differential systems [6]. The method will be reviewed below so as to make the present paper independent.

For bibliographical indications on the vast subject we refer to the papers quoted in the bibliography.

2. Summarization of the results. We shall say that a vector function $f(x, t), f=\left(f_{1}, \cdots, f_{n}\right)$, of the real vector $x=\left(x_{1}, \cdots, x_{N}\right)$, and of the real variable $t$ belongs to the class $A[\omega], \omega>0$, if for every $t$, $-\infty<t<+\infty$, each component $f_{j}$ of $f$ is analytic in a neighborhood $U$ of $x=(0, \cdots, 0)$ independent of $t$, and the power series expansion of $f_{j}$ in $x_{1}, \cdots, x_{N}$ is convergent in $U$, and its coefficients are periodic functions of $t$ of period $T=2 \pi / \omega$.

Presented to the Society, August 21, 1956; received by the editors July 19, 1956 and, in revised form, December 29, 1956. 
Consider the system of differential equations (1), or, in matrix form,

$$
\ddot{y}+D y=\epsilon f(y, \dot{y}, \epsilon ; t), \quad(\cdot=d / d t),
$$

where (a) $y=\left(y_{1}, \cdots, y_{n}\right), f=\left(f_{1}, \cdots, f_{n}\right), D=\operatorname{diag}\left(\sigma_{1}^{2}, \cdots, \sigma_{n}^{2}\right)$, (b) $\epsilon$ is real and $\sigma_{1}, \cdots, \sigma_{n}$ are positive numbers, (c) $f \in A[\omega]$, that is, for every $t, f_{j}$ is analytic in $y_{1}, \cdots, y_{n}, \dot{y}_{1}, \cdots, \dot{y}_{n}, \epsilon$ for $\left|y_{j}\right|<C$, $\left|\dot{y}_{j}\right|<C,|\epsilon|<\epsilon_{0}, j=1,2, \cdots, n$, where $C, \epsilon_{0}$ are independent of $t$, and the power series expansions, convergent for the same $y_{j}, \dot{y}_{j}, \epsilon$, have coefficients periodic in $t$ of period $T=2 \pi / \omega$.

Let $m$ be any integer, $0 \leqq m \leqq n$, and let $y=(u, w), u=\left(y_{1}, \cdots, y_{m}\right)$, $w=\left(y_{m+1}, \cdots, y_{n}\right), a=\left(a_{1}, \cdots, a_{n}\right), b=\left(b_{1}, \cdots, b_{n}\right), a_{j}, b_{j}$ positive integers, $c=\left(c_{1}, \cdots, c_{n}\right), \quad \sigma=\left(\sigma_{1}, \cdots, \sigma_{n}\right)$. Certain functions $H_{l}(a, b, c, \sigma, \omega, \epsilon)$, analytic in $\epsilon,|\epsilon|<\epsilon_{0}$, will be determined (\$4) for which the following theorem holds:

Theorem. If (A) $f_{j}(u,-w,-\dot{u}, \dot{w}, \epsilon,-t)=f_{j}(u, w, \dot{u}, \dot{w}, \epsilon, t)$, $j=1,2, \cdots, m$; (B) $f_{j}(u,-w,-\dot{u}, \dot{w}, \epsilon,-t)=-f_{j}(u, w, \dot{u}, \dot{w}, \epsilon, t)$, $j=m+1, \cdots, n$, and if, for $\epsilon$ sufficiently small, the system of equations

$$
\frac{a_{l}}{b_{l}} \omega+\epsilon H_{l}(a, b, c, \sigma, \omega, \epsilon)=\sigma_{l}, \quad l=1,2, \cdots, n,
$$

has a solution for some real nonzero $c$ and $\sigma$, then (1) has a solution $y(\epsilon, t) \in A\left[\omega / b_{1} \cdots b_{n}\right]$ with $y_{j}(0, t)=c_{j} \sigma_{j}^{-1} \cos \left(a_{j} \omega t / b_{j}\right), y_{j}(\epsilon,-t)$ $=y_{j}(\epsilon, t), j=1,2, \cdots, m, y_{j}(0, t)=c_{j} \sigma_{j}^{-1} \sin \left(a_{j} \omega t / b_{j}\right), y_{j}(\epsilon,-t)$ $=-y_{j}(\epsilon, t), j=m+1, \cdots, n$.

If $\sigma_{l}=a_{l} \omega / b_{l}$ and there exists a nonzero $c_{0}$ such that $H_{l}\left(a, b, c_{0}, \sigma\right.$, $\omega, 0)=0, l=1, \cdots, n$, and the determinant,

$$
\left|\partial H_{l}\left(a, b, c_{0}, \sigma, \omega, 0\right) / \partial c_{j}\right| \neq 0,
$$

then, for $\epsilon$ sufficiently small, system (2) certainly has a nonzero solution $c_{0}$.

It is interesting to note that condition (3) may be satisfied even in cases where the Jacobian obtained by applying Poincaré's periodicity condition [3] is identically zero. An example is given in [6, p. 389]. This example will be discussed in more detail in $\$ 5$.

The theorem above can also be considered as determining the numbers $\sigma_{j}$ when $a_{j}, b_{j}, c_{j}$ are given. Finally, the same theorem holds even when $f$ is independent of $t$ (autonomous case). Then system (2) determines relations between the basic "periods," $2 \pi b_{l} / a_{l} \omega$, of the dominant terms of the solutions and their "amplitudes," $c_{j}$. 
The theorem above for $m=n$ reduces to Theorems $(5.3, \mathrm{i})$ and (5.3, iii) of [6], and for $m=0$ to theorems $(5.3$, ii) and $(5.3$, iv) of [6]. by

It may be pointed out that the functions $H_{j}(a, b, c, \sigma, \omega, 0)$ are given

$$
\left\{\begin{aligned}
& H_{j}(a, b, c, \sigma, \omega, 0)=\frac{1}{c_{j} T} \int_{0}^{T} f_{j}\left(y_{0}, \dot{y}_{0}, 0, t\right) \cos \frac{a_{j}}{b_{j}} \omega t d t, \\
& j=1, \cdots, m, \\
& H_{j}(a, b, c, \sigma, \omega, 0)=\frac{1}{c_{j} T} \int_{0}^{T} f_{j}\left(y_{0}, \dot{y}_{0}, 0, t\right) \sin \frac{a_{j}}{b_{j}} \omega t d t, \\
& j=m+1, \cdots, n,
\end{aligned}\right.
$$

where $T=2 \pi b_{1} \cdots b_{n} / \omega$ and $y_{0}=\left(c_{1} \sigma_{1}^{-1} \cos a_{1} \omega t / b_{1}, \cdots, c_{m} \sigma_{m}^{-1}\right.$ $\left.\cdot \cos a_{m} \omega t / b_{m}, c_{m+1} \sigma_{m}^{-1} \sin a_{m+1} \omega t / b_{m+1}, \cdots, c_{n} \sigma_{n}^{-1} \sin a_{n} \omega t / b_{n}\right)$.

3. Review of the method. We will refer here, as in [6], to a system of first order ordinary equations of the form

$$
\dot{z}=A z+\epsilon q(z, \epsilon, t)
$$

where $A=\operatorname{diag}\left(\rho_{1}, \cdots, \rho_{N}\right), \rho_{1}, \cdots, \rho_{N}$, are complex numbers, $z=\left(z_{1}, \cdots, z_{N}\right), q=\left(q_{1}, \cdots, q_{N}\right)$, and $q \in A[\omega]$. First consider the auxiliary system

$$
\dot{z}=B z+\epsilon q(z, \epsilon, t),
$$

where $B=\operatorname{diag}\left(i \tau_{1}, \cdots, i \tau_{N}\right)$, and each $\tau_{j}$ is a rational multiple of $\omega$. Since $q \in A[\omega]$,

$$
q(z, \epsilon, t)=\sum_{k=0}^{\infty} \epsilon^{k} q^{(k)}(z, t), \quad q^{(k)}(z, t) \in A[\omega] .
$$

Let $s_{j}^{(r, k)}$ denote the coefficient of $\epsilon^{r-1}, r=1,2, \cdots$ when $z$ in $q_{j}^{(k)}(z, t)$ is replaced by $z=x^{(0)}(t)+\epsilon x^{(1)}(t)+\cdots$, where each $x^{(j)}(t)$ is independent of $\epsilon$. Moreover, let the corresponding coefficient of $\epsilon^{r-1}$ in $q_{j}(z, t, \epsilon)$ be denoted by $s_{j}^{(r)}$. Since only the case in which each $s_{j}^{(r, k)}$ is a periodic function of $t$ of some period $T$ will be considered, define

$$
\left\{\begin{aligned}
d_{j} S_{j}^{(r, k)} & =M\left[e^{-i \tau_{j} t} s_{j}^{(r, k)}\right] \\
d_{j} S_{j}^{(r)} & =M\left[e^{-i r_{j} t} s_{j}^{(r)}\right], \quad j=1,2, \cdots, N, \\
S^{(r)} & =\operatorname{diag}\left(S_{1}^{(r)}, \cdots, S_{N}^{(r)}\right),
\end{aligned}\right.
$$

where $d_{1}, \cdots, d_{N}$ are nonzero complex constants and $M[\cdot]$ denotes the ordinary mean value for periodic functions. It is clear that 


$$
\begin{aligned}
& s_{j}^{(r)}=s_{i}^{(r, 0)}+s_{j}^{(r-1,1)}+\cdots+s_{j}^{(1, r-1)}, \\
& S_{j}^{(r)}=S_{i}^{(r, 0)}+S_{j}^{(r-1,1)}+\cdots+S_{j}^{(1, r-1)}
\end{aligned}
$$

$$
j=1,2, \cdots, N .
$$

We now define the method of successive approximations as follows:

$$
\left\{\begin{array}{r}
z^{(0)}=x^{(0)}=\left(d_{1} e^{i \tau_{1} t}, \cdots, d_{N} e^{i \tau_{N} t}\right), \\
z^{(r)}=z^{(0)}+e^{B t} \int e^{-B t}\left[\sum_{k=1}^{r} \epsilon^{k} s^{(k)}-\left(\sum_{k=1}^{r} \epsilon^{k} S^{(k)}\right) z^{(r-1)}\right] d t \\
\left(\bmod \epsilon^{r+1}\right), r=1,2, \cdots,
\end{array}\right.
$$

where $s^{(k)}=\left(s_{1}^{(k)}, \cdots, s_{N}^{(k)}\right), z^{(r)}=x^{(0)}+\epsilon x^{(1)}+\cdots+\epsilon^{r} x^{(r)}, e^{B t}$ $=\operatorname{diag}\left(e^{i \tau_{1} t}, \cdots, e^{i \tau_{N} t}\right)$, and the above integrations are performed so as to obtain the unique primitive of mean value zero. It is clear that each $z^{(r)}$ defined by (9) is periodic in $t$, the period being determined by the numbers $\tau_{j}$. If we replace $z^{(r)}$ by its expression in terms of the $x^{(k)}$ and equate coefficients of powers of $\epsilon$, we obtain

$$
\left\{\begin{aligned}
x^{(0)}= & \left(d_{1} e^{i \tau_{1} t}, \cdots, d_{N} e^{i \tau_{N} t}\right), \\
x^{(r)=} & e^{B t} \int e^{-B t}\left[s^{(r)}-\left(S^{(1)} x^{(r-1)}+\cdots\right.\right. \\
& \left.\left.+S^{(r-1)} x^{(1)}\right) S^{(r)} x^{(0)}\right] d t \quad r=1,2, \cdots .
\end{aligned}\right.
$$

It is then shown in [6] that the method of successive approximations defined above converges to a solution of the system of equations

$$
\dot{z}=[B-\epsilon h(\tau, d, \epsilon)] z+\epsilon q(z, \epsilon, t),
$$

where $\tau=\left(\tau_{1}, \cdots, \tau_{N}\right), d=\left(d_{1}, \cdots, d_{N}\right), h=\operatorname{diag}\left(h_{1}, \cdots, h_{N}\right)$, and

$$
h(\tau, d, \epsilon)=S^{(1)}(d)+\epsilon S^{(2)}(\tau, d)+\epsilon^{2} S^{(3)}(\tau, d)+\cdots,
$$

where $S^{(r)}(\tau, d)$ is defined by (8). Consequently, the function $z$ satisfying (11) will be a solution of (5) if the system of equations

$$
i \tau_{k}-\epsilon h_{k}(\tau, d, \epsilon)=\rho_{k}, \quad k=1,2, \cdots, N,
$$

has a solution for some $\rho_{1}, \cdots, \rho_{N}$ and nonzero $d_{1}, \cdots, d_{N}$.

4. Proof of theorem. If, in (1), we make the transformations $y_{j}=v_{2 j-1}, \quad \dot{y}_{j}=v_{2 j}, \quad v_{2 j-1}=\left(2 i \sigma_{j}\right)^{-1}\left(z_{2 j-1}+z_{2 j}\right), \quad v_{2 j}=2^{-1}\left(z_{2 j-1}-z_{2 j}\right)$, then (1) is transformed into the system of first order equations

$$
\dot{z}=A z+\epsilon q(z, \epsilon, t),
$$

where $A=\operatorname{diag}\left(i \sigma_{1},-i \sigma_{1}, \cdots, i \sigma_{n},-i \sigma_{n}\right)$ and 


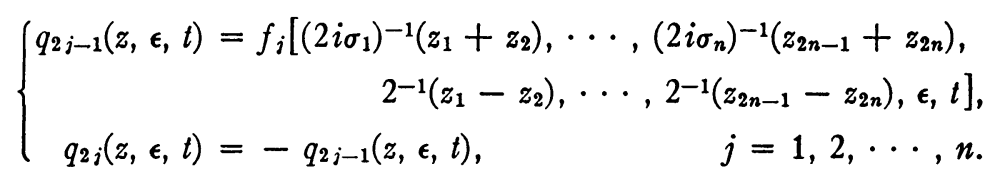

It is then shown in [6, pp. 365-368] that if the numbers $d_{k}, \tau_{k}$ are chosen so that $d_{2 j-1}=-\bar{d}_{2 j}$ ( ${ }^{-}$is the complex conjugate), $\tau_{2 j-1}=a_{j} \omega / b_{j}$, $\tau_{2 j}=-\tau_{2 j-1}$, where $a_{j}, b_{j}$ are positive integers, the function $z$ defined by the above algorithm (9) leads to real functions $v_{k}, k=1,2, \cdots, 2 n$, which are periodic in $t$ of period $T=2 \pi b_{1} \cdots b_{n} / \omega$. Furthermore, it is shown that $S_{2 j-1}^{(r)}=\bar{S}_{2 j}^{(r)}$ for every $r$ and, therefore, from (12), $h_{2 j-1}$ $=\bar{h}_{2 j}, j=1,2, \cdots, n$. Consequently, we will obtain a periodic solution of (1) if we can find nonzero numbers $d_{1}, d_{3}, \cdots, d_{2 n-1}$, $\sigma_{1}, \cdots, \sigma_{n}$ which satisfy the system of equations

$$
i a_{j} \omega / b_{j}-\epsilon h_{2-1}=i \sigma_{j}, \quad j=1,2, \cdots, n .
$$

In general, one can solve these $2 n$ equations ( $h_{2 j-1}$ may be complex) for combinations of the numbers $d_{1}, d_{3}, \cdots, d_{2 n-1}, \sigma_{1}, \cdots, \sigma_{n}$ to obtain periodic solutions of (1). However, to obtain solutions of the type specified in the theorem, further restrictions must be imposed on the numbers $d_{1}, d_{3}, \cdots, d_{2 n-1}$. We shall need the following lemma, which generalizes Lemmas $(5.2, \mathrm{i}),(5.2, \mathrm{ii})$ of [6].

Lemma. Suppose $f$ satisfies conditions (A) and (B) of the theorem. If we apply the preceding algorithm (9) to the auxiliary system of (14) with $d_{2 j-1}=i c_{j}, j=1,2, \cdots, m, d_{2 k-1}=c_{k}, k=m+1, \cdots, n$, where each $c_{l}, l=1,2, \cdots, n$ is a nonzero real number, then $x_{2 j-1}^{(r)}(-t)=x_{2 j}^{(r)}(t)$, $s_{2 j-1}^{(r)}(-t)=s_{2 j-1}^{(r)}(t), s_{2 j}^{(r)}(t)=-s_{2 j-1}^{(r)}(-t), s_{i j-1}^{(r)}=s_{2 j}^{(r)}, j=1,2, \cdots, m$, $x_{2 k-1}^{(r)}(-t)=-x_{2 k}^{(\tau)}(t), s_{2 k-1}^{(r)}(-t)=-s_{2 k-1}^{(\tau)}(t), s_{2 k}^{(\tau)}(t)=s_{2 k-1}^{(r)}(-t), s_{2 k-1}^{(r)}=s_{2 k}^{(r)}$, $k=m+1, \cdots, n, r=0,1,2, \cdots$, where the $x_{l}^{(r)}$ are defined by (10).

Proof. We shall first prove by induction that $x_{2 j-1}^{(r)}(-t)=x_{2 j}^{(r)}(t)$, $j=1,2, \cdots, m, x_{2 k-1}^{(r)}(-t)=-x_{2 k}^{(r)}(t), k=m+1, \cdots, n$ and all $r$. By our choice of the numbers $d_{l}$, the assertion is clearly true for $r=0$. Assume the assertion true for $r=0,1, \cdots, v-1$ and all $j, k$. Then $x_{2 j-1}^{(r)}(-t)+x_{2 j}^{(r)}(-t)=x_{2 j-1}^{(r)}(t)+x_{2 j}^{(r)}(t), \quad x_{2 j-1}^{(r)}(-t)-x_{2 j}^{(r)}(-t)$ $=-\left[x_{2 j-1}^{(r)}(t)-x_{2 j}^{(r)}(t)\right], j=1,2, \cdots, m, r=0,1, \cdots, v-1, x_{2 k-1}^{(r)}(-t)$ $+x_{2 \mathbf{k}}^{(r)}(-t)=-\left[x_{2 k-1}^{(r)}(t)+x_{2 \mathfrak{k}}^{(r)}(t)\right], x_{2 \mathfrak{k}-1}^{(r)}(-t)-x_{2 \mathfrak{k}}^{(r)}(-t)=x_{2 \mathfrak{k}-1}^{(r)}(t)-x_{2 \mathbf{k}}^{(r)}(t)$, $k=m+1, \cdots, n, r=0,1, \cdots, v-1$. Consequently, from conditions (A), (B), and formula (15), we have

$$
\left\{\begin{array}{r}
q_{2 j-1}\left[z^{(r)}(-t), \epsilon,-t\right]=q_{2 j-1}\left[z^{(r)}(t), \epsilon, t\right], \quad j=1,2, \cdots, m, \\
q_{2 k-1}\left[z^{(r)}(-t), \epsilon,-t\right]=-q_{2 k-1}\left[z^{(r)}(t), \epsilon, t\right], k=m+1, \cdots, n, \\
r=0,1,2, \cdots, v-1 .
\end{array}\right.
$$


Since $s_{2 j-1}^{(r+1)}(t)$ is the coefficient of $\epsilon^{r}$ in the expansion of $q_{2 j-1}\left(z^{(r)}, \epsilon, t\right)$, we have

$$
\left\{\begin{array}{lr}
(r) & \begin{array}{l}
(r) \\
s_{2 j-1}(-t)
\end{array}=s_{2 j-1}^{(r)}(t), \\
(r) & j=1,2, \cdots, m, \\
s_{2 k-1}(-t)=-s_{2 k-1}^{(r)}(t), & k=m+1, \cdots, n, r=1,2, \cdots, v .
\end{array}\right.
$$

Furthermore, since $q_{2 j-1}=-q_{2 j}$, it follows that $s_{2 j-1}^{(r)}=-s_{2 j}^{(r)}$ and

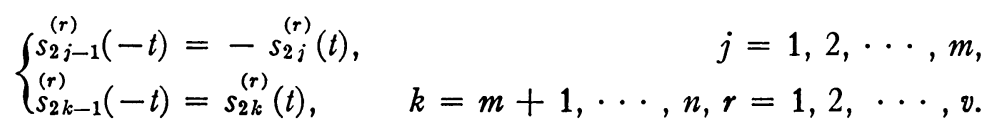

From the preceding relations we have

$$
\begin{aligned}
S_{2 j-1}^{(r)} & =\frac{1}{d_{2 j-1} T} \int_{0}^{T} e^{-i \tau_{2 j-1} t} s_{2 j-1}^{(r)}(t) d t \\
& =-\frac{1}{d_{2 j} T} \int_{0}^{T} e^{i \tau_{2 j} t} s_{2 j}^{(r)}(t) d t \\
& =\frac{1}{d_{2 j} T} \int_{0}^{-T} e^{-i \tau_{2 j} t} s_{2 j}^{(r)}(t) d t \\
& =-\frac{1}{d_{2 j} T} \int_{0}^{T} e^{-i \tau_{2 j} t} s_{2 j}^{(r)}(t) d t \\
& =-S_{2 j}^{(r)(r)}, \quad j=1,2, \cdots, m, r=1,2, \cdots, v .
\end{aligned}
$$

Similarly, $S_{2 k-1}^{(r)}=-S_{2 k}^{(r)}, k=m+1, \cdots, n, r=1,2, \cdots, v$. From (10),

$$
\begin{aligned}
x_{2 j-1}^{(v)}(-t)= & -e^{-i \tau_{2 j-1} t} \int e^{i \tau_{2 j-1} t}\left[s_{2 j-1}^{(v)}(-t)\right. \\
& \left.\quad-\left\{S_{2 j-1}^{(1)} x_{2 j-1}^{(v-1)}(-t)+\cdots+S_{2 j-1}^{(v)} x_{2 j-1}^{(o)}(-t)\right\}\right] d t \\
= & e^{i \tau_{2 j} t} \int e^{-i \tau_{2 j} t}\left[s_{2 j}^{(v)}(t)\right. \\
& \left.-\left\{S_{2 j}^{(1)} x_{2 j}^{(v-1)}(t)+\cdots+S_{2 j}^{(v)} x_{2 j}^{(o)}(t)\right\}\right] d t \\
= & j=1,2, \cdots, m .
\end{aligned}
$$

Similarly, $x_{2 k-1}^{(0)}(-t)=-x_{2 k}^{(0)}(t), k=m+1, \cdots, n$, and the induction on the $x_{l}^{(r)}$ is completed. If the assertion is true for $x_{l}^{(r)}$ for all $r$, then the other relations must also hold for all $r$ and the lemma is proved.

Using the above lemma and the fact that $S_{2 l-1}^{(r)}=\bar{S}_{2 l}^{(r)}$ for $l$ $=1,2, \cdots, n$ and all $r$, it follows that each $S_{2 l-1}^{(r)}$ and, therefore, from 
(12), each $h_{2 l-1}$ is purely imaginary. By using the above transformation formulas, it follows that the solution obtained in this manner has the properties mentioned in the Theorem. Furthermore, if we put $H_{l}(a, b, c, \sigma, \omega, \epsilon)=I\left(h_{2 l-1}\right), l=1,2, \cdots, n$ and apply (8), then the $H_{l}$ satisfy (4), and the theorem is proved. The details are as in [6, pp. 368, 375], and we refer to this paper for the sake of brevity.

5. Example. Consider the system of differential equations

$$
\begin{aligned}
& \ddot{x}+\sigma_{1}^{2} x=\epsilon \alpha x+\epsilon A \cos t \cdot x+\epsilon \beta x^{3}+\epsilon \gamma x y^{2}, \\
& \ddot{y}+\sigma_{2}^{2} y=\epsilon \delta y+\epsilon B \cos \omega t \cdot y+\epsilon \mu y^{3}+\epsilon \nu x^{2} y,
\end{aligned}
$$

where $\epsilon>0$ is a small parameter, and $\alpha, \beta, \gamma, \delta, \mu, \nu, A, B, \sigma_{1}, \sigma_{2}$ are real constants and $\omega$ is a rational number. This is the same as example 9.1 of [6]. We discuss this example again in order to show how more results may be obtained using the previous theorem.

Let us make the transformations in $\$ 4$ and apply the preceding algorithm to the auxiliary system of (20), taking the zeroth approximation to be $\left(a_{1} e^{i \tau_{1} t},-\bar{a}_{1} e^{-i \tau_{1} t}, a_{2} e^{i \tau_{2} t},-\bar{a}_{2} e^{-i \tau_{2} t}\right)$ where $\tau_{1}=k_{1} / m_{1}$, $\tau_{2}=k_{2} \omega / m_{2}$. Following the same discussion as in [6], it is easy to see that for $\tau_{1} \neq \tau_{2}, \tau_{1} \neq 1 / 2, \tau_{2} \neq \omega / 2$,

$$
\begin{aligned}
& S_{1}^{(1)}=\left(2 i \sigma_{1}\right)^{-1}\left[\alpha+3 \beta\left(4 \sigma_{1}^{2}\right)^{-1}\left|a_{1}\right|^{2}+\gamma\left(2 \sigma_{2}^{2}\right)^{-1}\left|a_{2}\right|^{2}\right], \\
& S_{3}^{(1)}=\left(2 i \sigma_{2}\right)^{-1}\left[\delta+\nu\left(2 \sigma_{1}^{2}\right)^{-1}\left|a_{1}\right|^{2}+3 \mu\left(4 \sigma_{2}^{2}\right)^{-1}\left|a_{2}\right|^{2}\right]
\end{aligned}
$$

and $S_{1}^{(1)}, S_{3}^{(1)}$ are purely imaginary for every $a_{1}, a_{2}$. Consequently, the classical Jacobian vanishes. However, since system (20) satisfies the conditions (A) and (B) for $m=0,1,2$, we know from the preceding lemma that for any $a_{1}, a_{2}$ purely imaginary, $a_{1}$ purely imaginary, $a_{2}$ real, or $a_{1}, a_{2}$ real, the real parts of the determining equations (16), $i \tau_{j}$ $-\epsilon S_{2 j-1}^{(1)}-\epsilon^{2} S_{2 j-1}^{(2)}-\cdots=i \sigma_{j}, j=1,2$, are identically zero and, thus, if $\sigma_{j}=\tau_{j}, j=1,2$, then the determining equations are $S_{1}^{(1)}+\epsilon S_{1}^{(2)}+\cdots$ $=0, S_{3}^{(1)}+\epsilon S_{3}^{(2)}+\cdots=0$. These equations have a real solution

$$
\left\{\begin{array}{l}
\left(\left|a_{1}\right| \sigma_{1}^{-1}\right)^{2}=-4(3 \mu \alpha-4 \gamma \delta)(9 \mu \beta-16 \gamma \nu)^{-1}+O(\epsilon), \\
\left(\left|a_{2}\right| \sigma_{2}^{-1}\right)^{2}=-4(3 \beta \delta-4 \nu \alpha)(9 \mu \beta-16 \gamma \nu)^{-1}+O(\epsilon),
\end{array}\right.
$$

provided that $\epsilon$ is sufficiently small and the right hand members of (21) are $>0$. This condition is certainly satisfied for some values of the constants in (20). If we interchange the two equations in (20), then conditions (A) and (B) are again satisfied for $m=1$. Thus, for $a_{2}$ purely imaginary and $a_{1}$ real, the conditions of the preceding lemma are satisfied and the equations (21) will have a solution for some 
$\alpha, \beta, \gamma, \delta, \mu, \nu$. Consequently, by the theorem, there are four different types of periodic solutions $(x, y)$ of $(20)$ each having the same amplitude for $\epsilon=0$; namely (i) $x, y$ even, (ii) $x$ even, $y$ odd (iii) $x$ odd, $y$ even, (iv) $x, y$ odd.

The exceptional cases $\tau_{1} \neq \tau_{2}, \tau_{1} \neq 1 / 2, \tau_{2} \neq \omega / 2$ may be treated in the same manner except (21) will contain the constants $A$ and $B$.

For the autonomous case, i.e., $A=B=0$, the preceding discussion applies if $\tau_{1}=k_{1} / m_{1}, \tau_{2}=k_{2} \omega / m_{2}, \tau_{1} \neq \tau_{2}, \omega$ rational and one obtains the same relations (21) for $\left|a_{1}\right|,\left|a_{2}\right|$. Therefore, for some values of $\alpha, \beta$, $\gamma, \delta, \mu, \nu$, and $A=B=0$, there are also four periodic solutions of (20) each having the same amplitude for $\epsilon=0$.

\section{BiBLIOGRAPHY}

1. L. Cesari, Sulla stabilita delle soluzioni dei sistemi di equazoni differenziali lineari a coefficienti periodici, Atti Accademia d'Italia. Memorie Classe Scienze Fisiche, Matematiche e Naturali (6) vol. 11 (1940) pp. 633-692.

2. L. Cesari and J. K. Hale, Second order linear differential systems with periodic L-integrable coefficients, Rivista Mat. Univ. Parma vol. 5 (1954) pp. 55-61.

3. E. A. Coddington and N. Levinson, Perturbation of linear systems with constant coefficients possessing periodic solutions, Annals of Math. Studies, Princeton University Press, vol. 29 (1952) pp. 19-35.

4. R. A. Gambill, Stability criteria for linear differential systems with periodic coefficients, Rivista Mat. Univ. Parma vol. 5 (1954) pp. 160-181.

5. - Criteria for parametric instability for linear differential systems with periodic coefficients, Rivista Mat. Univ. Parma vol. 6 (1955) pp. 37-43.

6. R. A. Gambill and J. K. Hale, Subharmonic and ultraharmonic solutions for weakly non-linear systems, Journal of Rational Mechanics and Analysis vol. 5 (1956) pp. 353-394.

7. J. K. Hale, Evaluations concerning products of exponential and periodic functions, Rivista Mat. Univ. Parma vol. 5 (1954) pp. 63-81.

8. - On boundedness of the solutions of linear differential systems with periodic coefficients, vol. 5 (1954) pp. 137-167.

9. - Periodic solutions of non-linear systems of differential equations, Rivista Mat. Univ. Parma vol. 5 (1954) pp. 281-311.

PURdUe UNIVERSITY AND

Remington Rand Univac, St. Paul, Minn. 